\title{
Quelques aspects démographiques de la population des femelles de la race bovine d'Aubrac
}

\author{
Jacqueline VU TIEN KHANG, B. BIBE et A. DALMIËRES \\ I.N.R.A., Station d'Amélioration génétique des Animaux \\ Centre de Recherches de Toulouse, B.P. 12, F 31320 Castanet-Tolosan
}

\section{Résumé}

Une analyse démographique a été réalisée sur un fichier retraçant, de 1970 à 1979, les carrières de 3102 vaches de la race d'Aubrac dans 49 élevages aveyronnais principalement conduits en race pure.

On a établi la structure par âge des reproductrices en 1979 : l'âge moyen estimé, 7 ans et 2 mois, confirme la bonne longévité de la vache Aubrac. Les tables de réforme des cohortes de mères 1970 à 1974 montrent que les éleveurs attendent généralement deux campagnes de production pour opérer une sélection.

L'examen du mouvement saisonnier des naissances indique une tendance à l'avancement en saison et au regroupement des naissances en période favorable.

Le rajeunissement observé de l'âge au premier vêlage traduit le désir des éleveurs de réduire la période improductive.

L'analyse de la distribution des intervalles entre vêlages successifs atteste leur volonté de ne conserver que des femelles vêlant régulièrement et de grouper les vêlages en période favorable. L'intervalle moyen entre vêlages, tous rangs confondus, calculé sur 1287 femelles ayant un premier vêlage à 3 ans, est égal à 371 jours, ce qui confirme la bonne fécondité des femelles Aubrac.

L'étude du renouvellement sur la voie mère-fille révèle l'existence d'une sélection des femelles sur ascendance maternelle chez 13 éleveurs traditionnellement sélectionneurs en race pure.

\section{Introduction}

Située sur la bordure sud-ouest du Massif Central, la région d'Aubrac est très variée à la fois d'un point de vue physique et économique (ANONYME, 1966 ; ANONYME, 1978). Malgré sa diversité, elle possède un puissant facteur d'unité : l'élevage bovin ; celui-ci exploite une population issue de la race locale d'Aubrac, bien adaptée aux conditions difficiles du milieu : période d'hivernage de longue durée avec restriction alimentaire et exploitation des montagnes en période estivale, à une altitude supérieure à 1000 mètres, sans abri et avec des déplacements importants (VISSAC, 1970; Valognes, 1972). Elevée dans un tel milieu, la race d'Aubrac a été sélec- 
tionnée sur ses qualités d'élevage : longévité, fécondité, aptitude au vêlage et à la mobilisation des réserves en période hivernale sans répercussion sur la reproduction (LILAS, 1980).

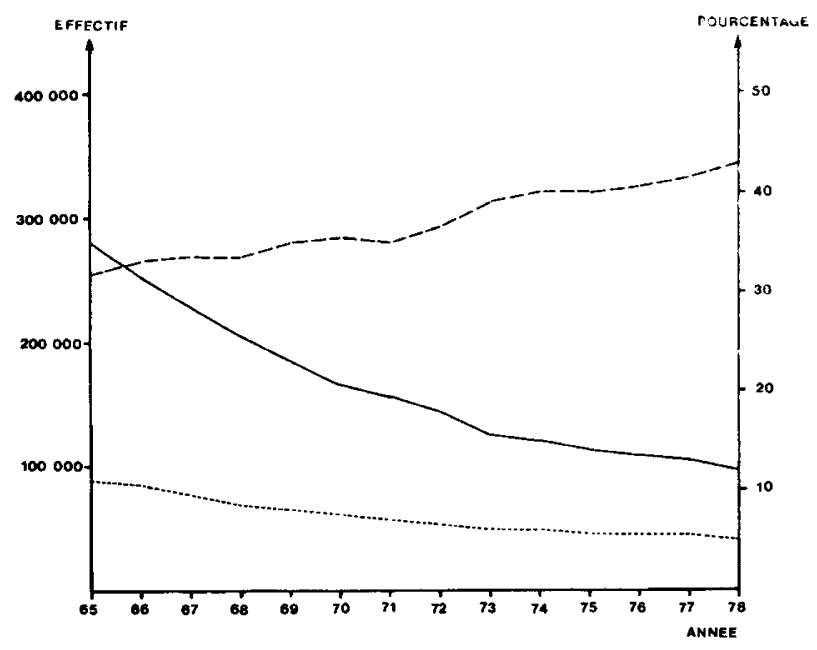

FIG. 1

Evolution comparée du cheptel Aubrac et du cheptel bovin total en Aveyron (animaux de plus de 6 mois).

Compared evolution of the Aubrac population and the whole cattle population in Aveyron (older than 6 months animals).

[Source : Services de la Fédération Départementale des Organismes de Défense Sanitaire.]

Effectif du cheptel Aubrac.

Size of the Aubrac population.

Effectif du cheptel total.

Size of the whole cattle population.

Pourcentage de cheptel Aubrac dans le cheptel total.

Percentage of the Aubrac cattle in the whole cattle population.

Après plusieurs tentatives infructueuses de croisement, l'introduction en 1959 du croisement Charolais $\times$ Aubrac a profondément modifié les systèmes de production, tout en faisant régresser l'effectif de la race d'Aubrac (fig. 1). En Aveyron, les vaches de race $A u b r a c$ ne représentaient plus, en 1979 , que 14 p. 100 du cheptel des mères (d'après le Service de Statistiques Agricoles et la Direction Départementale de l'Agriculture). Face à cette situation et conscients de l'intérêt que présente la femelle Aubrac, les éleveurs et leurs organismes se sont regroupés au sein de "l'Union $A u b r a c »(*)$ afin d'entreprendre un programme d'amélioration génétique destiné à

(*) L' «Union Aubrac», créée le 24 janvier 1979, comprend :

- le Herd Book de la race d'Aubrac (1 ${ }^{\text {er }}$ collège),

- l'union de sélection Midatest ( $2^{\mathrm{e}}$ collège)

- les Etablissements Départementaux de l'Elevage ayant des élevages de race Aubrac dans leur zone d'action, les groupements de producteurs et les Centres d'Insémination Artificielle (COOPEL-SO) ayant une activité en race d'Aubrac ( $3^{\text {e }}$ collège). 
relancer cette race. Son objectif est d'améliorer l'aptitude au croisement de la vache Aubrac avec des taureaux à fort développement musculaire, tout en lui conservant la faculté de bien se comporter dans son milieu (BIBÉ et al., 1974 ; VISSAC et al., 1976). Une des premières tâches de l'Union Aubrac a été de saisir sur support informatique les déclarations de naissance enregistrées depuis 1970 par les Etablissements Départementaux de l'Elevage de l'Aveyron, du Cantal et de la Lozère, ainsi que par les groupements de producteurs (Coopérative d'Elevage du Massif Central et Société d'Intérêt Collectif Agricole Montagne). C'est à partir d'un échantillon provenant de cet ensemble de données que nous avons abordé l'étude de quelques aspects démographiques de la population des femelles.

\section{Les données}

En 1979, l'Association Régionale de Service aux Organismes d'Elevage (A.R.S.O.E.) de Soual a saisi les déclarations de naissance, établies par les éleveurs, de tous les produits issus de mères de race $A u b r a c$ pendant la période allant de 1970 à 1979 (comprenant, en principe, les avortements et les morts-nés). Le regroupement des déclarations de naissance par numéro de mère a permis de reconstituer la carrière - ou, à défaut, la fraction connue de la carrière - de chaque mère et d'élaborer ainsi un fichier des carrières des vaches.

A partir de ce fichier général, nous avons créé un fichier de travail en extrayant les informations concernant les carrières des mères appartenant à 49 élevages situés en Aveyron (zone où l'information disponible présentait la meilleure qualité et, par ailleurs, département majoritaire en effectif de vaches Aubrac, devant la Lozère et le Cantal). Cet échantillon correspond aux élevages ayant établi des déclarations de naissance sans discontinuité de 1970 à 1979 et ne comprenant que des mères de race pure Aubrac; certains d'entre eux pratiquent le croisement mais ne conservent pas les produits croisés pour le renouvellement. Le mode de reproduction utilisé quasiexclusivement est la monte naturelle, sans synchronisation.

Ce fichier de travail a été vérifié et épuré. Deux catégories d'enregistrements ont été éliminées :

- celle des enregistrements relatifs à des mères ayant au moins un intervalle entre vêlages inférieur à 200 jours, causé par une déclaration de naissance affectée à tort à une femelle ;

- celle des enregistrements relatifs à des mères ayant au moins un intervalle entre vêlages supérieur à 730 jours. En effet, il est assez rare que les éleveurs gardent une femelle demeurant vide pendant une campagne $(*)$. Néanmoins, cette éventualité peut se produire, en particulier dans le cas de femelles ayant vêlé tardivement lors de la campagne précédente. Lorsque les éleveurs décident de conserver une femelle ayant «sauté » une campagne de reproduction, ils la remettent à la reproduction dès le début de la saison suivante : si elle ne «remplit» pas à ce moment, elle est alors réformée. C'est pourquoi les carrières comportant un intervalle entre vêlages

(*) La période de vêlage commençant avant le début de l'année civile, on a recours à la notion de campagne : par définition, la campagne $n$ est la période comprise entre le $1^{\text {er }}$ octobre de l'année civile $\mathrm{n}-1$ et le 30 septembre de l'année civile $n$. 
supérieur à 730 jours ont été éliminées du fichier : le choix de ce seuil permet d'écarter les carrières faussées par l'omission d'une déclaration de naissance, tout en minimisant le risque d'éliminer des carrières non erronées.

Environ 15 p. 100 des carrières ont ainsi été éliminées du fichier initial. Cette proportion n'est pas considérable, si l'on considère qu'un seul intervalle entre vêlages incohérent entraîne la suppression de toute la carrière de la femelle correspondante. Le fichier de travail concerne 3102 mères et 12923 produits. Il comprend deux types d'informations :

- des informations relatives aux mères, telles que leur numéro d'identification, leur numéro d'élevage, les numéros de leurs parents, leur date de naissance, leur nombre total de vêlages, le nombre total de leurs produits, leur âge au premier vêlage, les campagnes de début et de fin de leur carrière ;

- des informations relatives aux produits, telles que leur numéro d'identification, leur numéro d'élevage, les numéros de leurs parents, leur sexe, leur date de naissance, leur mode de naissance (gémellaire ou non), leur rang dans la fratrie, le laps de temps écoulé depuis la naissance du produit précédent.

\section{Critique des données}

Le fichier a été élaboré à partir des déclarations de naissance enregistrées de 1970 à 1979. De ce fait, on ignore la date de naissance des mères nées avant 1970 : elles sont regroupées dans une classe unique. En outre, on ne connaît rien du début de la carrière des femelles nées avant 1967, puisque seuls sont recensés les produits nés à partir de 1970 (la carrière reproductive des femelles commençant généralement à 3 ans).

Etant donnée la masse d'information saisie, les corrections n'ont pu être exhaustives. L'élimination des enregistrements aberrants, pratiquée lors de l'épuration du fichier, a soustrait de l'échantillon étudié un certain nombre d'animaux : ainsi, l'étude de l'évolution des effectifs par élevage est rendue impossible.

L'affectation automatique des mères à l'élevage dans lequel est né leur dernier produit interdit l'étude des mouvements de femelles en cours de production.

Enfin, l'élimination par les éleveurs d'une grande partie des femelles vides pendant une campagne et de la totalité des femelles vides pendant deux campagnes consécutives introduit un biais dans l'étude des intervalles entre vêlages.

\section{Structure par âge et table de réforme des reproductrices}

Nous considérons ici la population des reproductrices, c'est-à-dire des femelles ayant vêlé au moins une fois. La carrière de chaque mère est bornée par deux campagnes :

- sa campagne d'entrée dans la population, lors de sa naissance ;

- sa campagne de sortie de la population, lors de son dernier vêlage. 
Le traitement de cet ensemble de données, concernant les entrées et sorties. de femelles reproductrices, peut être conduit dans une perspective transversale, grâce au calcul de la structure par âge à une date fixée, ou dans une perspective longitudinale, grâce au calcul de la table de réforme de chaque cohorte (Pressat, 1973 et 1979).

\section{Structure par âge de la population des reproductrices}

Nous avons établi la structure par âge des reproductrices pendant la campagne 1979, de façon à disposer du maximum d'information sur l'âge des femelles présentes. Néanmoins, les déclarations de naissance n'ayant été enregistrées qu'à partir de 1970, les mères âgées de 10 ans (*) et plus ont dû être regroupées en une classe unique (tabl. 1 et fig. 2). Cette classe représente une fraction importante de l'effectif total (un peu plus de 20 p. 100), soit presque autant que les classes 7,8 et 9 ans. réunies.

\section{TABLEAU 1}

Structure par âge de la population des reproductrices en 1979. Age structure of the dams in 1979.

\begin{tabular}{c|c|c|c|c|c|c|c|c|c|c}
\hline $\begin{array}{c}\text { Classe d'âge } \\
\text { (années révolues) }\end{array}$ & 2 & 3 & 4 & 5 & 6 & 7 & 8 & 9 & $\begin{array}{c}10 \text { et } \\
\text { plus }\end{array}$ & Totat \\
\hline Nombre de mères & 19 & 293 & 293 & 258 & 185 & 140 & 142 & 119 & 369 & 1818 \\
Pourcentage .... & 1,05 & 16,12 & 16,12 & 14,19 & 10,18 & 7,70 & 7,81 & 6,55 & 20,30 & 100 \\
\hline
\end{tabular}

L'âge moyen des reproductrices de plus de 30 mois présentes en 1979 dans les. 49 élevages considérés a été estimé en faisant l'hypothèse d'une décroissance linéaire des effectifs de 10 à 17 ans. L'âge moyen obtenu, calculé sur 1799 mères, est de 7 ans et 2 mois : cette valeur confirme la bonne longévité de la vache Aubrac (FraYsSe, 1969).

\section{Tables de réforme de diverses cohortes de reproductrices}

Une cohorte est définie comme l'ensemble des reproductrices nées pendant une sampagne donnée. La table de réforme décrit la survenue, en fonction de l'âge, des sorties (morts, réformes, ventes) à l'intérieur d'une cohorte d'animaux. A partir d'un nombre arbitraire (égal ici à 1000 ) de femelles reproductrices présentes à l'âge de 3 ans (âge normal au premier vêlage), la table fournit, pour la suite des anniversaires $\mathbf{x}$ :

- le nombre $S_{x}$ de survivantes à l'anniversaire $x$;

- le nombre de sorties $\mathrm{d}(\mathrm{x}, \mathrm{x}+1)$ entre les deux anniversaires successifs $x$ et $x+1$ :

$$
d(x, x+1)=S_{x}-S_{x+1}
$$

${ }^{*}$ Les âges sont toujours exprimés en nombre d'années révolues. 


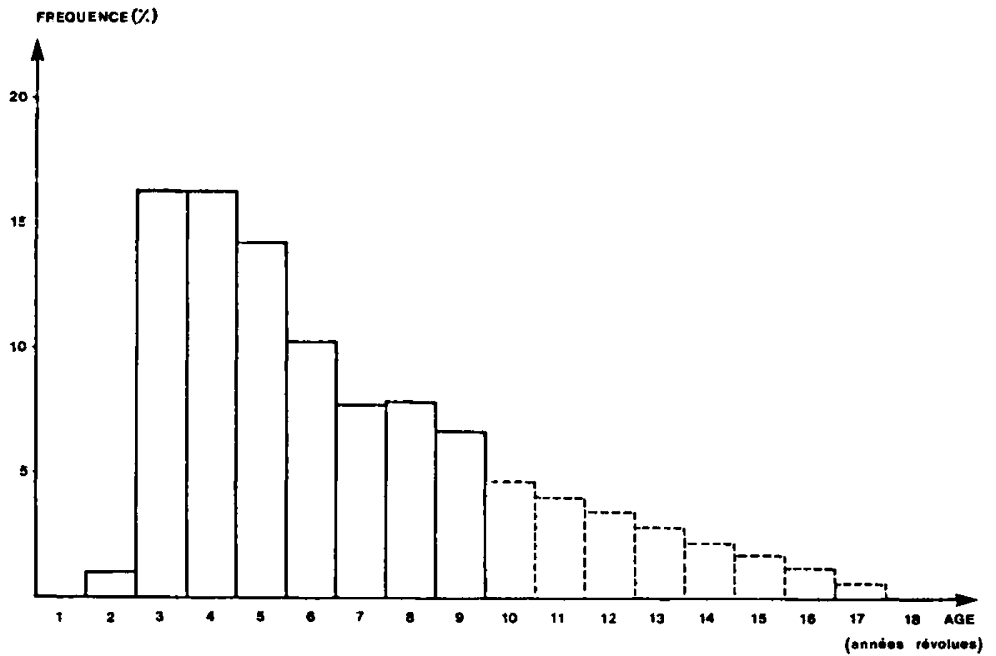

Fig. 2

Structure par âge de la populction des reproductrices en 1979 (la répartition des femelles âgées de plus de 10 ans a été faite en supposant une décroissance linéaire des effectifs de 10 à 17 ans).

$$
\text { Age structure of the dams in } 1979
$$

(over 10 years old females were distributed according to the assumption of a linear decrease of the size from 10 to 17 years).

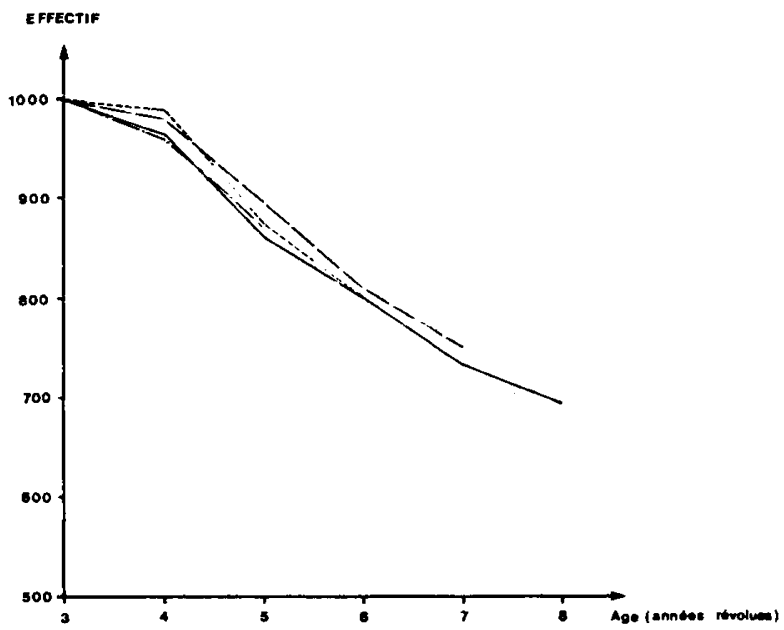

Fig. 3

Courbes de survie des cohortes de reproductrices 1970 à 1973.

Life curves of the dam cohorts from 1970 to 1973.

Cohorte 1970.

- - - - Cohorte 1971.

........... Cohorte 1972.

Cohorte 1973.

Cohorte $\mathrm{n}$ : ensemble des vaches nées pendant la campagne $\mathrm{n}$.

Cohort $n$ : cows born during annual period $n$. 
- le quotient de réforme $\mathrm{q}_{\mathrm{x}}$ à l'âge $\mathrm{x}$, égal à la probabilité, pour un individu présent à l'anniversaire $x$, de sortir de la population avant l'anniversaire $x+1$ :

$$
\mathrm{q}_{\mathrm{x}}=\frac{\mathrm{d}(\mathrm{x}, \mathrm{x}+1)}{\mathrm{S}_{\mathrm{x}}}=\frac{\mathrm{S}_{\mathrm{x}}-\mathrm{S}_{\mathrm{x}+1}}{\mathrm{~S}_{\mathrm{x}}}
$$

TABLEAU 2

Tables de réforme des cohortes de reproductrices 1970 à 1974.

Life tables of the dam cohorts 1970 to 1974.

\begin{tabular}{|c|c|c|c|c|c|c|c|}
\hline & \multirow{2}{*}{ Cohorte } & \multicolumn{6}{|c|}{ Age $x$ (en années révolues) } \\
\hline & & 3 & 4 & 5 & 6 & 7 & 8 \\
\hline 1970 & $\begin{array}{l}\mathrm{S}_{\mathrm{x}} \\
\mathrm{q}_{\mathrm{x}}(\%) \\
\mathrm{d}(\mathrm{x}, \mathrm{x}+1)\end{array}$ & $\begin{array}{c}1000 \\
3,37 \\
34\end{array}$ & $\begin{array}{l}956 \\
10,95 \\
106\end{array}$ & $\begin{array}{l}860 \\
7,26 \\
62\end{array}$ & $\begin{array}{l}798 \\
8,43 \\
67\end{array}$ & $\begin{array}{c}731 \\
5,26 \\
38\end{array}$ & 693 \\
\hline 1971 & $\begin{array}{l}\mathrm{S}_{\mathrm{x}} \\
\mathrm{q}_{\mathrm{x}}(\%) \\
\mathrm{d}(\mathrm{x}, \mathrm{x}+1)\end{array}$ & $\begin{array}{c}1000 \\
2,23 \\
22\end{array}$ & $\begin{array}{l}978 \\
8,48 \\
83\end{array}$ & $\begin{array}{c}895 \\
9,76 \\
87\end{array}$ & $\begin{array}{l}808 \\
7,03 \\
57\end{array}$ & 751 & \\
\hline 1972 & $\begin{array}{l}\mathrm{S}_{\mathrm{x}} \\
\mathrm{q}_{\mathrm{x}}(\%) \\
\mathrm{d}(\mathrm{x}, \mathrm{x}+1)\end{array}$ & $\begin{array}{c}1000 \\
1,00 \\
10\end{array}$ & $\begin{array}{l}990 \\
11,51 \\
114\end{array}$ & $\begin{array}{c}876 \\
8,47 \\
74\end{array}$ & 802 & & \\
\hline 1973 & $\begin{array}{l}S_{x} \\
q_{x}(\%) \\
d(x, x+1)\end{array}$ & $\begin{array}{c}1000 \\
4,00 \\
40\end{array}$ & $\begin{array}{l}960 \\
9,28 \\
89\end{array}$ & 871 & & & \\
\hline 1974 & $\begin{array}{l}\mathrm{S}_{\mathrm{x}} \\
\mathrm{q}_{\mathrm{x}}(\%) \\
\mathrm{d}(\mathrm{x}, \mathrm{x}+1)\end{array}$ & $\begin{array}{c}1000 \\
0,67 \\
7\end{array}$ & 993 & & & & \\
\hline \multicolumn{8}{|c|}{$\begin{array}{l}\mathrm{S}_{\mathrm{x}}=\text { nombre de survivantes à l'anniversaire } \mathrm{x} \text {. } \\
S_{x}=\text { number of survivors at xth birthday. } \\
\mathrm{d}(\mathrm{x}, \mathrm{x}+1)=\mathrm{S}_{\mathrm{x}}-\mathrm{S}_{\mathrm{x}+1}\end{array}$} \\
\hline
\end{tabular}

Les tables de réforme ont été établies pour les cohortes 1970 à 1974 (tabl. 2). L'absence de disparité entre les diverses cohortes a été vérifiée par des tests d'homogénéité du $X^{2}$ réalisés sur le tableau des effectifs bruts observés. Les figures 3 et 4 présentent les courbes de survie et de réforme des cohortes considérées. Ces données. se rapportent à des vaches ayant vêlé au moins une fois : si elles ne permettent pas d'appréhender la sélection effectuée avant le début de la carrière reproductive (par élimination des génisses infécondes, notamment), elles fournissent, en revanche, des indications sur la politique de sélection pratiquée par les éleveurs vis-à-vis des repro- 
ductrices. L'élimination entre 3 et 4 ans, c'est-à-dire après le premier vêlage, est faible (environ 2 p. 100). Le quotient de réforme augmente à 4 ans, où il atteint 11 p. 100 en moyenne, puis il diminue régulièrement jusqu'à l'âge de 7 ans. Les éleveurs attendent donc deux campagnes de production avant d'effectuer une sélection parmi les reproductrices. Ceci pourrait s'expliquer par la pratique d'une sélection individuelle des femelles sur leur premier intervalle entre vêlages. De plus, les éleveurs attendent peut-être que les vaches aient réalisé un deuxième allaitement pour opérer une sélection à la fois sur leurs performances de reproduction et sur leurs performances laitières.

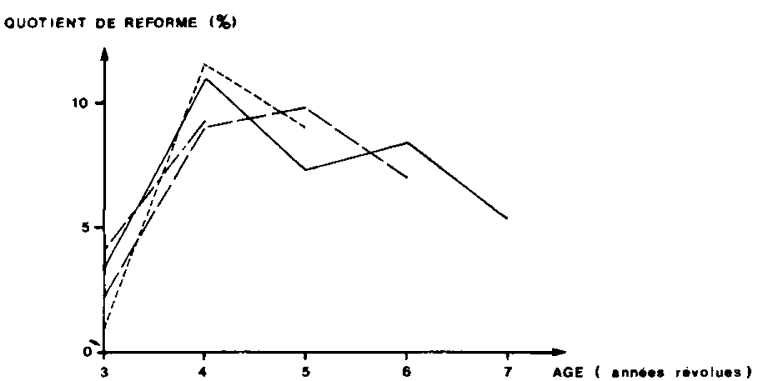

Fig. 4

Quotients de réforme des cohortes de reproductrices 1970 à 1973.

Reform quotients of the dam cohorts from 1970 to 1973.

\section{Cohorte 1970.}

Cohorte 1971.

_............. Cohorte 1972.

Cohorte $\mathrm{n}$ : ensemble des vaches nées pendant la campagne $\mathrm{n}$.

Cohort $n$ : cows born during annual period $n$.

L'information disponible sur la carrière des femelles étant tronquée en 1979, il n'est pas possible de suivre les cohortes jusqu'à leur extinction ni, par conséquent, de calculer leur espérance de vie.

\section{La reproduction}

\section{Age au premier vêlage}

Dans la race d'Aubrac, les vêlages avant 30 mois sont relativemení rares : selon les cohortes, ils représentent 4 à 9 p. 100 de l'ensemble des premiers vêlages. L'âge moyen à la mise bas des femelles vêlant avant 30 mois est assez stable : il oscille autour de 2 ans et 2 mois. 
En revanche, on observe un net rajeunissement des femelles vêlant pour la première fois après 30 mois : leur âge moyen passe de 3 ans 1 mois et 15 jours dans la cohorte 1970, à 2 ans 11 mois et 27 jours dans la cohorte 1976, baissant ainsi de 49 jours. Ce déplacement de la moyenne est accompagné d'un resserrement de la distribution et d'une réduction de l'écart-type (tabl. 3, fig. 5). Ce rajeunissement de l'âge au premier vêlage traduit l'intérêt que portent les éleveurs à la réduction de la période improductive.

TABLEAU 3

Age au premier vêlage des mères ayant vêlé après 30 mois. Age at first calving of dams having their first calving after 30 months.

\begin{tabular}{|c|c|c|c|c|c|c|c|c|}
\hline Cohorte de mères & 1970 & 1971 & 1972 & 1973 & 1974 & 1975 & 1976 & $\begin{array}{c}\text { En- } \\
\text { semble } \\
.1970 \\
\text { à } 1976\end{array}$ \\
\hline Age moyen (jours) & 1140 & 1122 & 1114 & 1108 & 1095 & 1104 & 1091 & 1108 \\
\hline Ecart-type (jours) & 130 & 1.23 & 105 & 104 & 90 & 87 & 46 & 99 \\
\hline$\ldots \ldots \ldots$ & 180 & 200 & 179 & 217 & 266 & 270 & 263 & 1575 \\
\hline
\end{tabular}

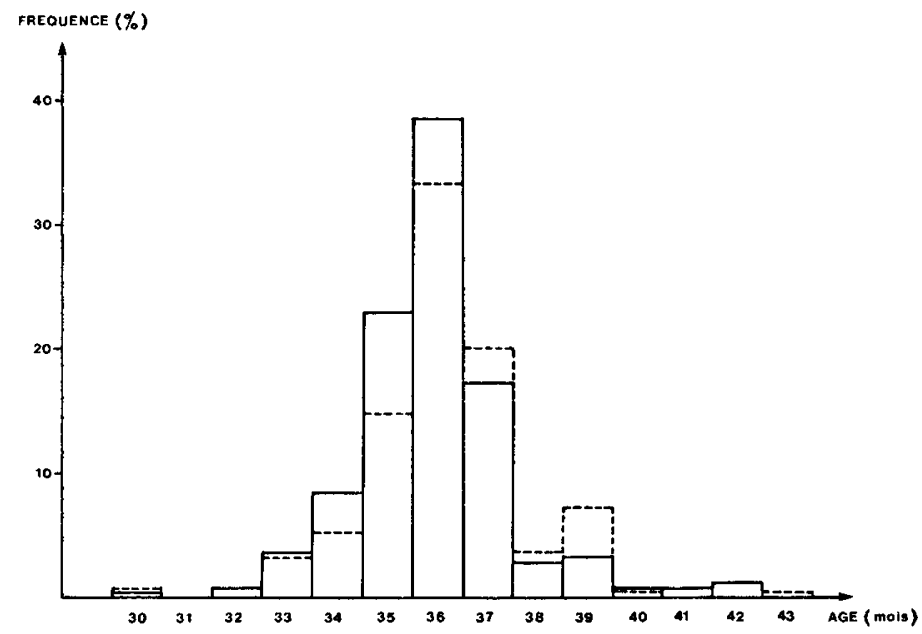

FIG. 5

Histogramme des âges au premier vêlage des cohortes 1970 et 1976 (exception faite des femelles ayant vêlé à 2 ans).

Histogram of the ages at first calving for the cohorts 1970 and 1976 (excluding dams having calved at 2 years). 


\section{Mouvement saisonnier des naissances}

L'étude de la distribution saisonnière des dates de naissance revêt un intérêt particulier en troupeau de vaches allaitantes, car on admet classiquement l'existence d'une période favorable de vêlage, réalisant la meilleure adéquation entre les besoins du troupeau et les disponibilités fourragères (JARRIGE, 1979). En région d'Aubrac, cette époque couvre les mois de janvier à mars. Pour des raisons de commodité de calcul et compte tenu du faible nombre de vêlages pendant les mois d'octobre à décembre, nous l'avons étendue du $1^{\text {er }}$ octobre au 31 mars.

Dans cette étude, nous traiterons séparément le cas des produits issus des quelques génisses vêlant avant 30 mois : ces femelles ont un calendrier de reproduction tout à fait distinct des autres. En effet, les quelques produits issus de vêlage avant 30 mois naissent tardivement et le plus souvent hors de la période favorable (tabl. 4). Le vêlage à deux ans est un phénomène limité (cf. paragraphe $\mathrm{V}-1$ ) qui résulterait plutôt de saillies accidentelles survenues en estive que d'une réelle volonté des éleveurs : la date moyenne de ces vêlages, qui se situe généralement en mai, confirme cette hypothèse.

\section{TABLEAU 4}

Date de naissance des produits issus de premiers vêlages à 2 ans (campagnes 1972 à 1979).

Birthday of the calves born from first calvings at 2 years (annual periods 1972 to 1979).

\begin{tabular}{|c|c|c|c|c|c|c|c|c|c|}
\hline Campagne & 1972 & 1973 & 1974 & 1975 & 1976 & 1977 & 1978 & 1979 & \begin{tabular}{|c} 
En- \\
semb'e \\
(1972 à \\
$1979)$
\end{tabular} \\
\hline $\begin{array}{l}\text { Date moyenne } . \\
\text { (jour/mois) }\end{array}$ & $15 / 05$ & $c i s / 05$ & $29 / 04$ & $12 / 05$ & $23 / 05$ & $\therefore 8 / 05$ & $07 / 04$ & $18 / 05$ & $09 / 05$ \\
\hline $\begin{array}{l}\text { Ecart-type } \\
\text { (jours) }\end{array}$ & 64,9 & 46,2 & 63,4 & 53,9 & 49,9 & 37,5 & 44,1 & 30,9 & 42,8 \\
\hline$\%$ NPF (1) $\ldots$. & 33,3 & 15,4 & 36,8 & 26,9 & 6,9 & 15,8 & 42,9 & 5,3 & 21,2 \\
\hline Effectif & 12 & 13 & 19 & 26 & 29 & 19 & 14 & 19 & 151 \\
\hline
\end{tabular}

(1) $\%$ NPF = pourcentage de naissances en période favorable (du 1-10 au 31-3).

$\%$ NPF $=$ percentage of births in the favourable period (from October 1st to March 31 th).

De manière générale, le premier vêlage n'intervient pas avant l'âge de 30 mois. Les primipares vêlent en moyenne plus tôt en saison que les autres (fig. 6, tabl. 5 et 6), l'écart entre ces deux catégories variant selon les campagnes (de 3 jours en 1974 et 1977 , à 10 jours en 1978). Dans l'ensemble, les dates moyennes des naissances ont tendance à devenir plus précoces. Entre les campagnes 1974 et 1979, elles passent : 

mipares ;

- du 26 février au 5 mars (7 jours d'écart) pour les produits issus de pri-

- du 2 mars au 8 mars (6 jours d'écart) pour les autres.

\section{TABLEAU 5}

Date de naissance des produits issus de premiers vêlages à 3 ans (campagnes 1974 à 1979).

Birthday of the calves born from first calvings at 3 years (annual periods 1974 to 1979).

\begin{tabular}{|c|c|c|c|c|c|c|c|}
\hline Campagne & 1974 & 1975 & 1976 & 1977 & 1978 & 1979 & $\begin{array}{c}\text { Ensemble } \\
\text { (1974 à } \\
1979)\end{array}$ \\
\hline $\begin{array}{l}\text { Date moyenne .. } \\
\text { (jour/mois) }\end{array}$ & $05 / 03$ & $05 / 03$ & $02 / 03$ & $27 / 02$ & $21 / 02$ & $26 / 02$ & $28 / 02$ \\
\hline Ecart-type (jours) & 47,2 & 41,9 & 39,8 & 36,0 & 33,6 & 39,5 & 39,7 \\
\hline$\%$ NPF (1) & 80,8 & 81,9 & 83,9 & 83,4 & 85,7 & 85,8 & 83,8 \\
\hline Effectif & 208 & 188 & 217 & 271 & 266 & 281 & 1431 \\
\hline
\end{tabular}

(1) $\%$ NPF $=$ pourcentage de naissances en période favorable (du 1-10 au 31-3).

$\% N P F=$ percentage of births in the favourable period (from October 1st to March 31th).

\section{TABleau 6}

Date de naissance des produits issus de vêlages de rang supérieur à 1

(campagnes 1974 à 1979).

Birthday of the calves born from higher rank calvings

(annual periods 1974 to 1979).

\begin{tabular}{|c|c|c|c|c|c|c|c|}
\hline Campagne & 1974 & 1975 & 1976 & 1977 & 1978 & 1979 & $\begin{array}{c}\text { Ensemble } \\
(1974 \text { à } \\
1979)\end{array}$ \\
\hline $\begin{array}{l}\text { Date moyenne } . . \\
\text { (jour/mois) }\end{array}$ & $\mathrm{Cs} / 03$ & $09 / 03$ & $07 / 03$ & $02 / 03$ & $02 / 03$ & $02 / 03$ & $04 / 03$ \\
\hline Ecart-type (jours) & 41,3 & 44,6 & 40,2 & 40,1 & 37,2 & 34,0 & 38,4 \\
\hline$\%$ NPF (1) $\ldots$ & 75,4 & 78,8 & 78,5 & 84,0 & 81,0 & 84,0 & 81,6 \\
\hline Effectif $\ldots \ldots \ldots$ & 195 & 401 & 578 & 780 & 1015 & 1161 & 4130 \\
\hline
\end{tabular}

(1) $\%$ NPF $=$ pourcentage de naissances en période favorable (du 1-10 au 31-3).

$\%$ NPF $=$ percentage of births in the favourable period (from October 1st to March 31 th). 
Simultanément, on note une tendance au regroupement des naissances : l'écarttype de la distribution tend à se réduire. Cette évolution s'accompagne de l'augmentation du pourcentage de naissances en période favorable (tabl. 5 et 6). Mais il faut signaler que ces valeurs moyennes recouvrent en fait une grande disparité entre élevages : la proportion de naissances en période favorable varie de 50 p. 100 à 95 p. 100 selon les élevages.

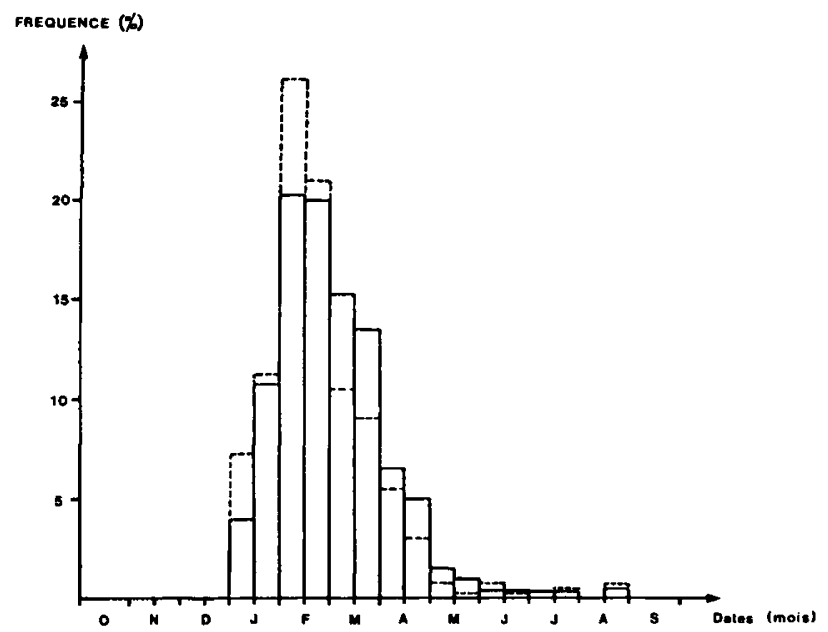

Fig. 6

Distribution saisonnière des premiers vêlages et des vêlages de rang supérieur à 1 pendant la campagne 1979 (les femelles ayant vêlé à 2 ans sont exclues).

Seasonal distribution of the first calvings and of the other rank calvings during the annual period 1979 (excluding dams having calved at 2 years).

Premiers vêlages.

First calvings.

Vêlages de rang supérieur à 1 .

Other calvings.

\section{Intervalle entre vêlages}

Dans l'étude des intervalles entre vêlages successifs, nous considérons séparément les mères vêlant avant 30 mois. En effet, elles vêlent le plus souvent hors de la période favorable (cf. paragraphe $\mathrm{V}-1$ ) et, de ce fait, sont soumises à un rythme particulier de reproduction.

Cas des femelles vêlant pour la première fois avant 30 mois

Le premier vêlage avant 30 mois représentant un cas de figure minoritaire, nous devons procéder à des regroupements de cohortes afin de pouvoir raisonner sur des effectifs suffisants. La figure 7 présente la courbe de distribution du premier intervalle entre vêlages chez les 119 femelles des cohortes 1970 à 1975 ayant vêlé avant 30 mois et, à titre de référence, la courbe équivalente chez les 1287 femelles 


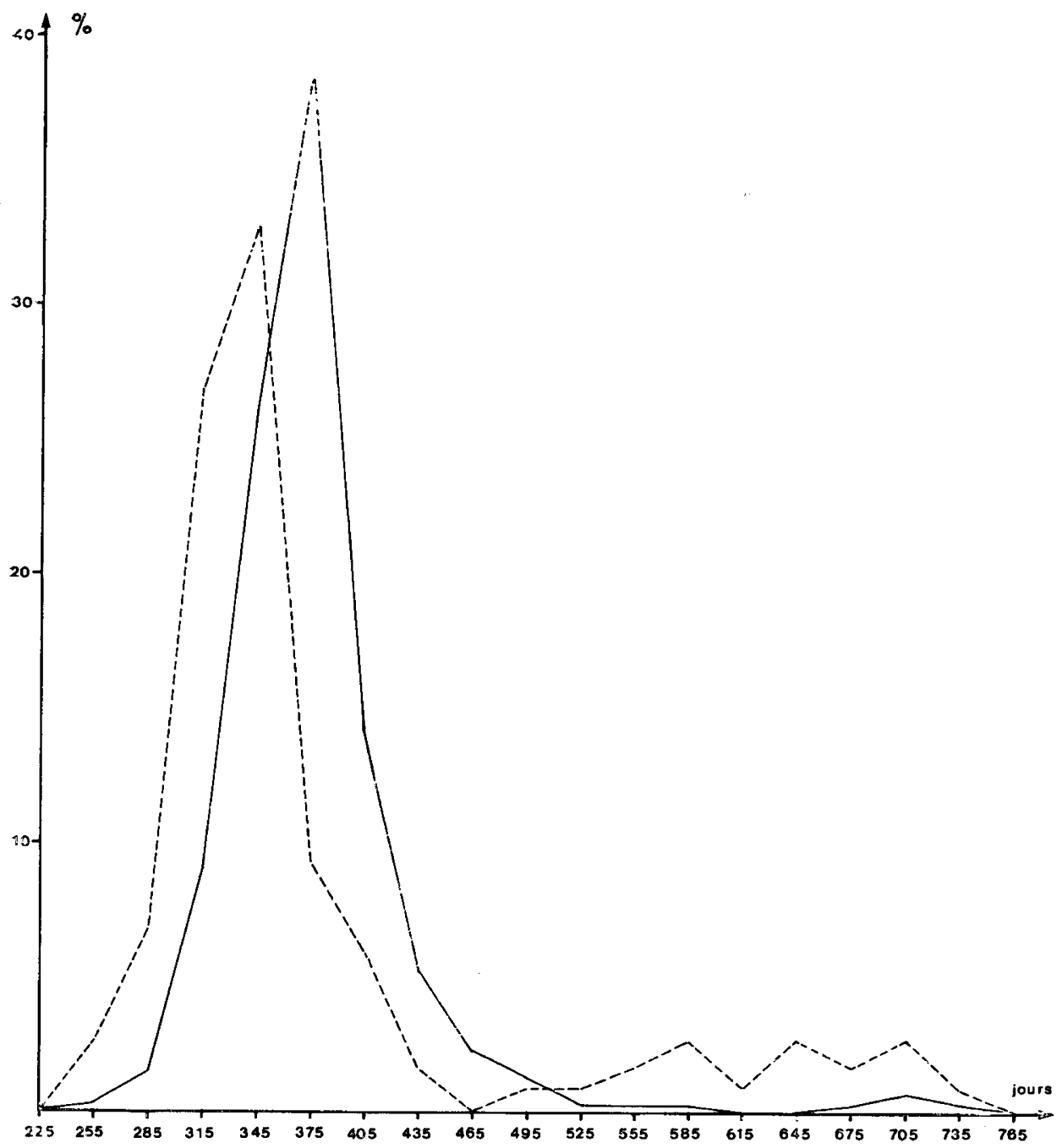

FIG. 7

Distribution du premier intervalle entre vêlages chez les femelles des cohortes 1970 à 1975 . Distribution of the first calving interval of the 1970 to 1975 cohorts.

Femelles ayant eu leur premier vêlage avant 30 mois. Dams having first calved before 30 months.

Femelles ayant eu leur premier vêlage après 30 mois. Dams having first calved after 30 months.

(Les nombres de jours figurant sur l'échelle des abscisses représentent les valeurs centrales de classes de 30 jours : par exemple le point 345 correspond à la classe 330-360 jours). (The numbers of days on the absciss axis represent the central values of 30 days classes: for instance, the point 345 accounts for the class 330 to 360 days). 
des mêmes cohortes ayant eu leur premier vêlage après 30 mois. Alors que les premiers intervalles entre vêlages des femelles ayant eu leur premier vêlage après 30 mois se distribuent de façon assez regroupée et symétrique autour de la classe modale (360-390 jours), ceux des femelles ayant vêlé avant 30 mois se répartissent en deux catégories :

1) 86 p. 100 d'entre eux, inférieurs à 450 jours, se distribuent autour de la classe modale (330-360 jours) ;

2) 14 p. 100 d'entre eux sont supérieurs à 450 jours : ce sont ces longs intervalles qui «gonflent» la moyenne générale (378 jours, contre 375 jours chez les femelles ayant eu leur premier vêlage après 30 mois).

On peut donner à ces observations l'interprétation suivante : les femelles qui ont vêlé avant 30 mois ont eu ce premier vêlage assez tardivement en saison (cf. paragraphe V-1). Les éleveurs tentent de les rapprocher du calendrier normal de reproduction en les remettant rapidement à la saillie. En cas de réussite, leur premier intervalle entre vêlages est, en moyenne, inférieur à celui du reste de la population. En cas d'échec, les éleveurs leur font sauter une saison de reproduction : un long intervalle s'écoule alors entre le premier et le deuxième vêlage.

Les intervalles moyens entre vêlages de rang 2-3 (calculé sur les cohortes 1970 à 1975) et 3-4 (calculé sur les cohortes 1970 à 1974) sont plus courts chez les femelles ayant commencé leur carrière reproductive avant 30 mois que dans le reste de la population : ils sont respectivement de 356 jours (sur un effectif de 96) contre 370 jours (sur un effectif de 902) et de 358 jours (sur un effectif de 68) contre 368 jours (sur un effectif de 612). Ces écarts s'estompent dans les intervalles ultérieurs (4-5 et suivants). Ces observations s'expliquent vraisemblablement par le désir des éleveurs de remettre progressivement «en phase» des femelles ayant commencé leur carrière reproductive avec un net décalage par rapport au reste du troupeau.

\section{Cas des femelles vêlant pour la première fois après 30 mois}

Les femelles vêlant pour la première fois après 30 mois ont un premier intervalle moyen entre vêlages de 375 jours. Contrairement aux femelles vêlant à 2 ans, elles n'ont un premier intervalle supérieur à 450 jours que dans environ 5 p. 100 des cas (fig. 7). Ces femelles ont donc tendance à décaler légèrement leur date de vêlage entre le premier et le second, ce qui justifie la pratique consistant à faire vêler les primipares plus tôt en saison que le reste du troupeau (cf. paragraphe $\mathrm{V}-1$ ). Ce premier intervalle, légèrement plus long que les suivants (tabl. 7), pourrait s'expliquer par une répercussion de l'alimentation sur la reproduction. En effet, les primipares reçoivent en général la même ration que les autres vaches, alors que leurs besoins sont supérieurs, leur croissance n'étant pas terminée.

Le tableau 7 fournit les moyennes et les écarts-types des intervalles entre vêlages de rangs successifs dans les cohortes de mères 1970 à 1975 ayant eu leur premier vêlage à 3 ans; la figure 8 présente la distribution de ces intervalles dans la cohorte 1970. Il faut toutefois remarquer que les échantillons regroupant les derniers intervalles connus de chaque cohorte risquent d'être légèrement tronqués du fait de l'arrêt de l'observation à la fin de 1979 (les femelles ayant eu un long intervalle entre vêlages échappant à l'observation). 


\section{TABLEAU 7}

Intervalle entre vêlages en fonction du rang de vêlage, pour les cohortes de mères 1970 à 1975 ayant eu leur premier vêlage à 3 ans.

Calving intervals according to the calving rank, for the dams of 1970 to 1975 cohorts having their first calving at 3 years.

\begin{tabular}{|c|c|c|c|c|c|c|c|}
\hline \multirow{2}{*}{$\begin{array}{l}\text { Intervalles } \\
\text { entre vêlages }\end{array}$} & \multicolumn{7}{|c|}{ Cohortes de mères } \\
\hline & Paramètres & 1970 & 1971 & 1972 & 1973 & 1974 & 1975 \\
\hline \multirow{3}{*}{ IVV $1-2(1) \ldots$} & Moyenne & 377,6 & 377,1 & 380,3 & 371,9 & 373,9 & 371,9 \\
\hline & Ecart-type & 57,3 & 66,9 & 56 & 59,4 & 45 & 34,9 \\
\hline & Effectif & 177 & 196 & 178 & 215 & 264 & 257 \\
\hline \multirow{3}{*}{ IVV $2-3$} & Moyenne & 376,2 & 375,2 & 366,7 & 370,3 & 362,7 & \\
\hline & Ecart-type & 66,9 & 66,3 & 52,3 & 49,7 & 37,6 & \\
\hline & Effectif & 157 & 175 & 157 & 192 & 221 & \\
\hline \multirow{3}{*}{ IVV $3-4$} & Moyenne & 369,3 & 368,7 & 363,7 & 370,2 & & \\
\hline & Ecart-type & 60,6 & 56,2 & 44,9 & 40,6 & & \\
\hline & Effectif & 146 & 164 & 144 & 158 & & \\
\hline \multirow{3}{*}{ IVV $4-5 \ldots \ldots$} & Moyenne & 362,9 & 371,4 & 367,6 & & & \\
\hline & Ecart-type & 34 & 32,8 & 34,3 & & & \\
\hline & Effectif & 135 & 147 & 117 & & & \\
\hline \multirow{3}{*}{ IVV 5-6 } & Moyenne & 364,3 & 366,6 & & & & \\
\hline & Ecart-type & 30,6 & 30,9 & & & & \\
\hline & Effectif & 124 & 113 & & & & \\
\hline \multirow{3}{*}{ IVV 6.7} & Moyenne & 375,9 & & & & & \\
\hline & Ecart-type & 42,3 & & & & & \\
\hline & Effectif & 94 & & & & & \\
\hline
\end{tabular}

(1) IVV $n-(n+1)=$ intervalle entre les nième et $(n+1)$ ième vêlages. IVV $n-(n+1)=$ interval between the $n^{\text {th }}$ and the $(n+1)$ th calving.

Considérons la distribution des intervalles entre vêlages successifs dans la cohorte 1970, qui est celle dont la longueur de carrière connue est la plus grande (fig. 8) : on constate que les valeurs observées sont bien regroupées autour des deux classes modales (330-360 jours et 360-390 jours). La proportion d'intervalles supérieurs à 450 jours est faible : elle oscille entre les valeurs extrêmes de 0,74 p. 100 (intervalle entre $4^{\prime \prime}$ et $5^{\circ}$ vêlage) et 7,01 p. 100 (intervalle entre $2^{\circ}$ et $3^{\text {e }}$ vêlage). 


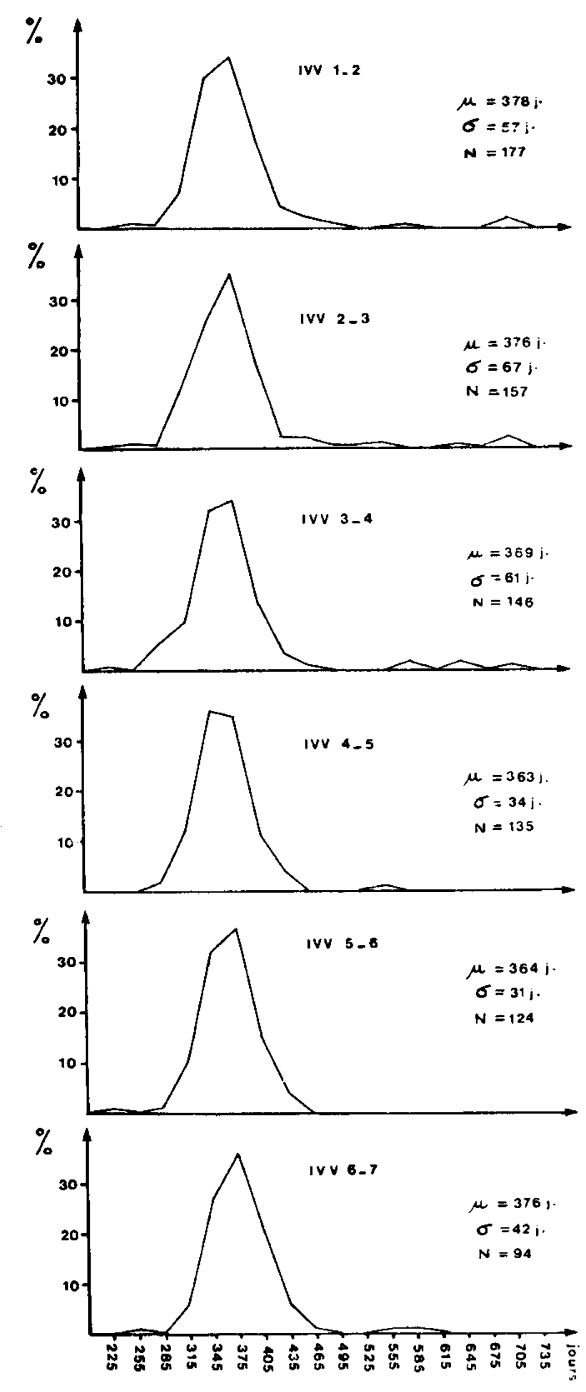

Fig. 8

Distribution des intervalles entre vêlages successifs chez les femelles de la cohorte 1970 ayant eu leur premier vêlage après 30 mois.

Distribution of the successive calving intervals for the dams of the 1970 cohort having their first calving after 30 months.

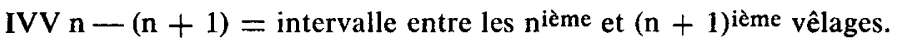

$\mu=$ moyenne (jours).

$\sigma=$ écart-type (jours).

$\mathbf{N}=$ effectif de l'échantillon.

IVV $n-(n+1)=$ interval between the $n^{\text {th }}$ and the $(n+1)^{\text {th }}$ calving.

$\mu=$ mean (days).

$\sigma=$ standard deviation (days).

$\mathbf{N}=$ size of the sample.

(L'échelle des abscisses est la même que sur la fig. 7).

(The absciss scale is the same as on fig. 7). 
Pour un rang fixé d'intervalles entre vêlages, on note une tendance à la réduction de l'écart-type au fil des cohortes successives (tabl. 7) : ce résultat confirme les progrès réguliers réalisés par les éleveurs dans la maîtrise du calendrier de reproduction.

L'intervalle moyen entre vêlages, tous rangs confondus, pour les 1287 femelles des cohortes 1970 à 1975 ayant un premier vêlage après 30 mois, est égal à 371 jours. Si l'on considère que les quotients de réforme observés en cours de carrière ne sont pas très importants (plus de 70 p. 100 des reproductrices d'une cohorte donnée sont encore présentes dans le troupeau à l'âge de 8 ans), cette valeur traduit une bonne fécondité de la femelle Aubrac. Etant donné les conditions de milieu dans lesquelles sont exploitées les vaches $A$ ubrac (restriction alimentaire en période hivernale et mise au taureau généralement tardive, vers avril-mai), la durée de gestation (283 jours, d'après CAMINADE, 1975) et le long anoestrus de lactation couramment observé chez les vaches allaitantes, cette valeur représente en effet une bonne performance.

\section{Prolificité}

Sur les 12681 vêlages connus des 3102 vaches du fichier, on observe un taux moyen de prolificité de 101,9 p. 100. Cette valeur est légèrement inférieure à celle calculée par Lienard et LEgENDRE (1974) sur 2207 femelles Aubrac pendant les campagnes 1969 et $1970: 102,8$ p. 100.

A titre de comparaison, les résultats fournis par LIENARd et LEgENDRE (1974) sont de :

- 102,5 p. 100 pour la Salers (3 341 femelles dont 632 génisses),

- 100,4 p. 100 pour la Limousine (1 411 femelles dont 239 génisses),

- 104,2 p. 100 pour la Charolaise (8 772 femelles dont 1621 génisses).

\section{Le renouvellement}

Notre fichier de travail étant un fichier de mères, la seule voie de renouvellement que nous puissions étudier est la voie mère-fille. Dans cette étude, nous traiterons de façon distincte :

- un «noyau» de 13 élevages traditionnellement sélectionneurs en race pure (groupe I) ;

- l'ensemble des 36 autres élevages, qui pratiquent le croisement à un degré plus ou moins important (groupe II).

Parmi l'ensemble des femelles qui naissent dans un troupeau, certaines sont conservées pour la reproduction : ce sont ces filles qui, devenant à leur tour des reproductrices, assurent le renouvellement du troupeau. Nous convenons d'appeler.

- $n_{i j}$ le nombre total de filles nées, pendant la campagne $j$, de mères appartenant à la cohorte $\mathrm{i}$;

- $m_{i j}$ le nombre de filles qui, parmi les $n_{i j}$ nées pendant la campagne $j$ de mères appartenant à la cohorte $\mathrm{i}$, deviennent des reproductrices dans le troupeau ;

- $r_{i j}$ le rapport de $m_{i j}$ à $n_{i j}$. 
L'étude du renouvellement de la population des reproductrices peut être abordée dans une perspective longitudinale (à partir d'une cohorte de mères), ou transversale (à partir d'une cohorte de filles).

\section{Approche longitudinale}

Considérons la cohorte de mères nées pendant la campagne 1970 : c'est celle dont la partie connue de la carrière est la plus longue. Sur cette partie connue de carrière, la proportion $r_{70, j}$ de filles conservées pour la reproduction parmi l'ensemble des filles nées pendant la campagne $\mathrm{j}$, culmine en 1975 , à l'âge de 5 ans (c'est-à-dire à la $3^{\mathrm{e}}$ campagne de production) dans les élevages du groupe $I$ et en 1973, à l'âge de 3 ans (c'est-à-dire à la $1^{\text {re }}$ campagne de production) dans les élevages du groupe II (fig. 9).

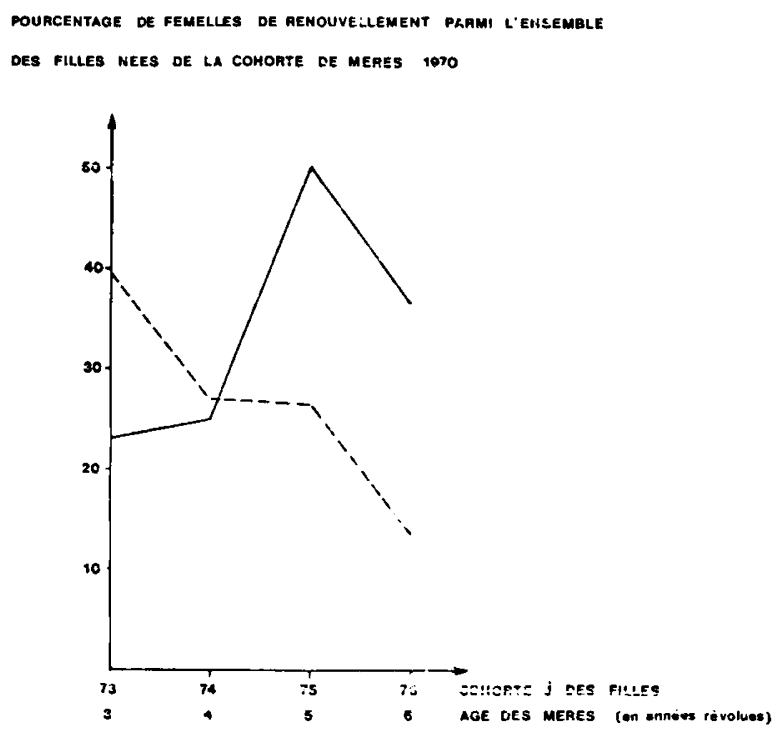

Fig. 9

Participation de la cohorte de mères 1970 à la procréation des diverses cohortes de femelles de renouvellement.

Participation of the 1970 dam cohort in the procreation of the different replacement female cohorts.

Groupe I (13 élevages sélectionneurs en race pure).

Group I (13 breeders' herds).

. . . . . . . . Groupe II (36 autres élevages).

Group II (36 other herds).

Les éleveurs sélectionneurs du noyau des 13 élevages du groupe I manifestent une tendance à conserver des femelles issues de mères dont la carrière est connue : ces résultats attestent l'existence, chez ce noyau d'éleveurs, d'une sélection des femelles sur ascendance maternelle. Une telle sélection est beaucoup plus difficile à 
réaliser dans un élevage pratiquant le croisement. En effet, les éleveurs évitent généralement d'accoupler les jeunes femelles de race Aubrac avec des taureaux Charolais, en raison des risques de dystocie (qui sont plus élevés au premier vêlage que lors des vêlages ultérieurs). Ils choisissent plutôt de pratiquer un premier vêlage en race pure et de conserver les femelles issues de ce vêlage pour le renouvellement. Cette contrainte, inhérente au système d'élevage, compromet la possibilité de sélection des femelles sur ascendance maternelle.

\section{Approche transversale}

Considérons la cohorte de filles nées pendant la campagne 1976 : c'est la cohorte la plus récente dans laquelle nous connaissions le devenir des femelles (conservation en vue de la reproduction ou élimination). La figure 10 présente, dans chacun des deux groupes d'élevages considérés, la proportion de femelles de renouvellement dans la cohorte de filles nées en 1976, en fonction de l'âge de leurs mères. On constate tout d'abord que les éleveurs en race pure (groupe I) conservent une proportion plus importante de génisses de renouvellement (la sélection ultérieure sur les femelles en production étant plus forte dans ce groupe). D'autre part, on n'observe pas de grandes disparités dans la participation des diverses cohortes de mères au renouvellement. Dans le groupe $I$, ce sont les mères âgées de 6 ans qui produisent les filles ayant la plus forte probabilité de devenir reproductrices. Dans le groupe II, ce sont les mères âgées de 3 ans qui possèdent cette caractéristique. Ces résultats confirment la différence de conduite déjà observée entre les deux groupes d'élevages lors de l'étude longitudinale.

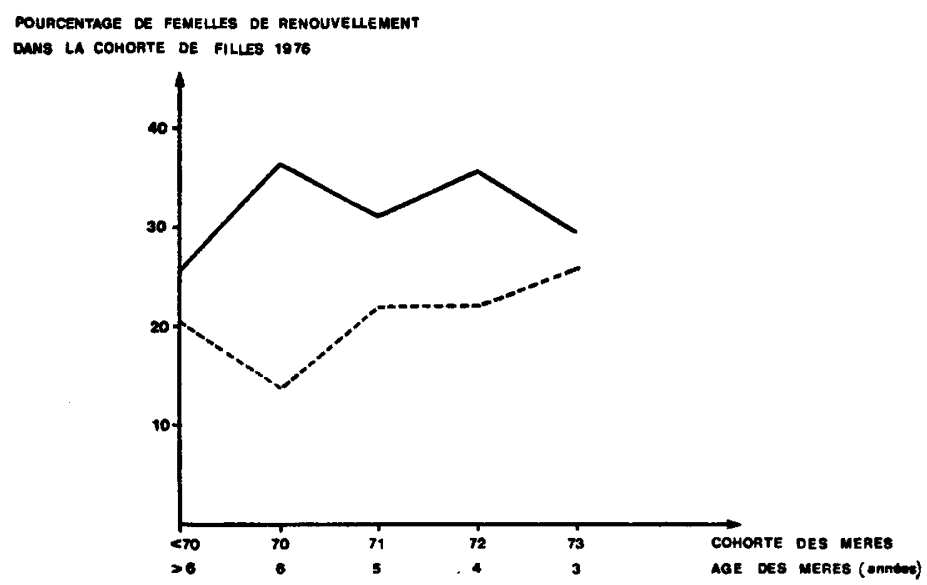

Fig. 10

Proportion de femelles de renouvellement dans la cohorte de filles 1976 en fonction de l'âge de leurs mères.

Proportion of replacement females in the 1976 daughter cohort according to their mothers' age.

Groupe I (13 élevages sélectionneurs en race pure).

Group I (13 breeders' herds).

Group II (36 autres élevages).

Group 11 (36 other herds). 
Le regroupement en une classe unique des mères nées avant 1970 rend impossible le calcul de l'intervalle de génération sur la voie mère-fille.

\section{Taux de renouvellement et de réforme de la population des reproductrices}

Le taux de renouvellement est défini comme le rapport du nombre de primipares (c'est-à-dire de femelles entrant dans la population des reproductrices) au nombre total de reproductrices présentes au début de la campagne. Le taux obtenu pour la campagne 1979 est de 17,9 p. 100, soit 318 primipares pour 1777 mères présentes en début de campagne.

Le taux de réforme est défini comme le rapport du nombre de mères éliminées durant une campagne au nombre total de reproductrices présentes au début de la campagne. Le taux obtenu pour la campagne 1979 est de 15,25 p. 100, soit 271 mères éliminées sur 1777 mères présentes en début de campagne. Cette valeur est nettement supérieure aux quotients de réforme observés dans diverses cohortes (tabl. 2, fig. 4). Cette apparente discordance s'explique de la façon suivante : le taux de réforme est calculé à partir des reproductrices de tous âges présentes à un moment donné, alors que les quotients de réforme du tableau 2 se rapportent à des âges variant de 3 à 7 ans. A la décroissance continue du quotient de réforme observée à partir de 4 ans, succède vraisemblablement une forte remontée due aux réformes de femelles âgées : ceci expliquerait le taux global de réforme calculé sur l'ensemble des reproductrices.

Le calcul des taux de renouvellement et de réforme n'a pas été effectué pour les campagnes antérieures à 1979, car les éliminations d'animaux pratiquées lors de l'épuration du fichier auraient conduit à des résultats erronés.

\section{Conclusion}

Cette approche démographique de la population des femelles de race Aubrac permet de dégager quelques caractéristiques et de détecter certaines tendances.

La volonté des éleveurs de grouper les vêlages en période favorable constitue une contrainte importante du système d'élevage et influe fortement sur le rythme de la reproduction. Ainsi, le premier vêlage après 30 mois est la règle générale (les vêlages plus précoces se situant le plus souvent hors de la période favorable). D'autre part, la date moyenne du premier vêlage est un peu plus précoce en saison que celle des vêlages ultérieurs, compte tenu du fait que le premier intervalle entre vêlages est en moyenne un peu plus long que les suivants. Cette volonté de grouper les vêlages pendant la période favorable est attestée par l'évolution, observée sur les dix dernières années, des paramètres de la distribution des dates de naissance : avancement en saison de la date moyenne et réduction de l'écart-type. Ces modifications tendent à augmenter le pourcentage de naissances en période favorable.

Certains résultats de l'étude démographique fournissent des éléments d'information sur la politique de sélection des femelles. L'élimination des femelles infécondes et de celles ayant un trop long intervalle entre vêlages (qui explique en grande partie les quotients de réforme observés au début de la carrière reproductive) constitue une sélection individuelle. Cette sélection résulte de l'attachement des éleveurs aux caractères de reproduction et de leur volonté de grouper les vêlages en période 
favorable. En outre, le sous-groupe des éleveurs sélectionneurs en race pure utilise la connaissance de la. carrière maternelle comme critère de choix des femelles à conserver pour la reproduction : il pratique une sélection des femelles sur ascendance maternelle.

Les valeurs obtenues pour l'intervalle moyen entre vêlages et pour l'âge moyen des mères, qui confirment la bonne fécondité et la bonne longévité de la vache Aubrac, traduisent son adaptation aux conditions de milieu dans lesquelles elle est exploitée.

Reçu pour publication en mars 1982.

\section{Remerciements}

Les auteurs remercient l'A.R.S.O.E. de Soual, les E.D.E. de l'Aveyron, du Cantal et de la Lozère, ainsi que l'Union Aubrac, sans lesquels cette étude n'aurait pu être conduite.

Ils remercient $H$. LERIDON (Institut National d'Etudes Démographiques) ainsi que Annick Gibon et F. MÉNISSIER (I.N.R.A., Département de Génétique animale) pour leurs critiques et suggestions lors de la lecture du manuscrit.

\section{Summary \\ Some demographic characteristics of the female population of the Aubrac cattle breed}

The reproductive life of 3102 cows of the Aubrac breed (a local mountain breed of the South Massif Central) has been demographically analysed. Data studied were collected, from 1970 to 1979, from 49 herds located in Aveyron and mainly in pure breeding.

Age structure of dams is tabulated. Estimated average age is found to be 7 years and 2 months : this figure confirms the good longevity of the Aubrac cow. Life tables of dams were constructed for the cohorts 1970 to 1974 : it appears that breeders usually begin culling only after two years of production.

Seasonality of calving shows a tendancy toward earlier births which are more concentrated in the favourable period for beef production in this country (winter).

Earlier age at first calving indicates that breeders wish to shorten length of non-productive life.

Distribution of the successive calving intervals is indicative of their purpose to keep only regularly calving females and to group calving in the favourable period. Average calving interval has been computed on every rank for a sample of 1287 females having their first calving at 3 years : the figure obtained (371 days) confirms the good fecundity of $A u b r a c$ cows.

The analysis of the replacement of female stock points out, in the 13 traditionally breeders' herds, a selection on dam's value.

\section{Références bibliographiques}

ANONYME, 1966. L'Aubrac, tome I : étude ethnologique, linguistique, agronomique et économique d'un établissement humain. C.N.R.S., Paris, 300 p.

ANONYME, 1978. Production de viande dans le Nord-Aveyron : dix ans d'évolution. Rapport du C.D.A.N.A.-E.D.E., 50 p. 
BibÉ B., Frebling J., Ménissier F., 1974. Schéma d'utilisation des races rustiques en croisement avec des races à viande. In $\mathrm{VI}^{\mathrm{es}}$ Journées d'Informations du Grenier de Theix : « L'exploitation des troupeaux de vaches allaitantes $»$, Supplément technique du CRZV de Theix, Numéro spécial, octobre 1974. I.N.R.A.-I.T.E.B., 192-211.

Caminade J.L., 1975. La race bovine Blonde d'Aquitaine : essai de méthodologie pour la classification des femelles. Mémoire de fin d'études, Ec. Sup. Agric. de Purpan, Toulouse, $230 \mathrm{p}$.

Fraysse J.L., 1969. Recherche en race Aubrac de souches ayant les meilleures qualités d'élevage à la lumière des problèmes que pose le milieu naturel. Mémoire de fín d'études, Ec. Nat. Ing. Trav. Agric., Bordeaux, 88 p.

JARRIGE R., 1979. Utilisation des pâturages des milieux défavorisés : essai de conclusions. In $\mathrm{X}^{\circ:}$ Journées du Grenier de Theix : "Utilisation par les ruminants des pâturages d'altitude et parcours méditerranéens », I.N.R.A., Versailles, 541-565.

Lienard G., Legendre J., 1974. Productivité en veaux des troupeaux de vaches allaitantes. In VI"* Journées d'Informations du Grenier de Theix : "L'exploitation des troupeaux de vaches allaitantes », Supplément technique du CRZV de Theix, Numéro spécial, octobre 1974. I.N.R.A.-I.T.E.B., 47-67.

LILAS J.P., 1980. Approche pour une meilleure connaissance de la race d'Aubrac. Mémoire de fin d'études, Ec. Nat. Sup. Agric. Appli., Dijon, 73 p.

Pressat R., 1973. L'analyse démographique. P.U.F., Paris, 321 p.

Pressat R., 1979. Dictionnaire de démographie. P.U.F., Paris, 295 p.

VALOGNES R., 1972. Evolution des exploitations et des productions bovines dans l'Aubrac. Mémoire de fin d'études, Ec. Nat. Sup. Sci. Agric. Appli., Dijon, 40 p.

Vissac B., 1970. Etude génétique de la race d'Aubrac. In L'Aubrac, tome I, C.N.R.S., Paris, 27-101.

Vissac B., Bibé B., Frebling J., Menissier F., Casu S., Boyazoglu J.G., 1976. Potentialités des populations bovines locales en élevage extensif dans les zones montagneuses et méditerranéennes. Options méditerr., 35, 76-90. 